\title{
Osseointegration of hydroxyapatite coatings doped with silver nanoparticles: scanning electron microscopy studies on a rabbit model
}

\author{
Ł. Łapaj, W. Woźniak, J. Markuszewski \\ Department of General Orthopaedics, Musculoskeletal Oncology, Trauma Surgery, \\ Poznan University of Medical Sciences, Poznan, Poland
}

[Received: 25 March 2018; Accepted: 23 May 2018]

Background: Modern joint arthroplasties rely on osseointegration of metal components through bone ingrowth into hydroxyapatite (HA) layers. However, such surfaces are prone to colonisation by bacteria and formation of biofilms. Application of silver nanoparticles (SNs) to hydroxyapatite coatings could reduce the risk of infection; however, little is known about how this would affect the process of bone ingrowth. This study examined osseointegration of conventional and SN doped HA coatings in a rabbit model.

Materials and methods: In this study, 12 cylindrical implants coated with conventional and SN doped HA were implanted into New Zealand white rabbit femora, with each animal receiving both types of implants. After 12 weeks, rabbits were sacrificed, their femora were harvested and implants removed during pull-out testing. Retrieved samples were dehydrated, sputter coated and observed using a scanning electron microscope (SEM) to verify bony ingrowth and retention of SNs. Results: The percentage of implant in direct contact with bone was measured in cross-sections of implants. The SEM analysis demonstrated that osseointegration of the SN doped coatings was similar to the conventional HA samples. A similar morphology of newly formed trabecular bone was observed in both implants, with silver doped HA-coated implants retaining multiple nanoparticles in areas which were not overgrown by bone. Analysis of the bone-implant contact area revealed comparable results for both types of coatings. These finding indicated that $S N$ doped HA coatings are characterised by good osseointegrative properties. Conclusions: Since SNs were found in areas not covered by mineralised bone, it is assumed that the antimicrobial properties of the modified coating may be retained for 12 weeks after implantation. Additional studies are required to fine--tune the composition of HA coatings with SNs, to ensure optimal osseointegrative and antimicrobial properties. (Folia Morphol 2019; 78, 1: 107-113)

Key words: bone remodelling, orthopaedic coatings, bone formation

\section{INTRODUCTION}

Long term performance of joint arthroplasties heavily depends on stable anchoring of implants in bone $[9,20]$. Since the bone-implant interface is subjected to cyclic loading during physical activities, insufficient mechanical fixation results in implant migration and loosening $[9,20]$. Consequently, modern uncemented joint replacements rely on biologic fixation through

Address for correspondence: Dr. Ł. Łapaj, Department of General Orthopaedics, Musculoskeletal Oncology, Trauma Surgery; Poznan University of Medical Sciences, ul. 28 Czerwca 1956 roku nr 135/147, 61-545 Poznań, Poland, tel/fax: + 486183 10 164, e-mail: llapaj@orsk.ump.edu.pl 
ingrowth of bone into various substrates, such as porous titanium or hydroxyapatite (HA) coatings $[12,15]$.

Although multiple studies demonstrated good long term results of uncemented arthroplasties, several failure modes of such components have been identified. Periprosthetic joint infections (PJIs) are responsible for a large percentage of such failures, in both early and late follow-up [20]. This is due to the fact that materials used in joint replacements may become colonised by biofilm forming bacteria $[2,6]$. The biofilm can act as a layer which encapsulates bacteria and protects them from the host immune response and antibiotics. Consequently, management of PJls typically involves surgical exchange of contaminated components and long term antibiotic therapy [20].

Since PJIs are associated with dissatisfactory clinical outcomes and a significant financial burden, there is a need for the development of biofilm inhibiting materials for use in total joint replacements $[4,8,21]$. Electrochemically formed silver coatings were one of early solutions used in resection prostheses since early 2000s [11, 21]. In conventional uncemented components such coatings may be problematic, due to concerns regarding osseointegration and silver toxicity; however, silver nanoparticles (SNs) have recently emerged as an alternative $[14,21]$. They can be distributed on the surface of uncemented components without compromising the osseointegrative layer [6]. Multiple laboratory studies have shown their antimicrobial potential, while data from animal models has indicated good biocompatibility $[3,4,8]$.

Still, specific interactions between host bone and coatings containing SNs are not well understood. The effect of SNs on osseointegration of orthopaedic implants is of particular interest, since it is critical for their long term performance $[2-4,7,8,15,21]$. This study examined the osseointegration of SN doped HA coatings in a rabbit model. The samples were obtained after mechanical testing of implant stability (pull-out tests), which allowed the visualisation of areas where newly bone was firmly attached to the HA coatings. This study determined if the presence of silver (Ag) nanoparticles affects the microstructure of newly formed bone and verified if SNs become resorbed during osseointegration.

\section{MATERIALS AND METHODS}

\section{Study design}

This experimental study compared osseointegration of two types of implants in femoral bones of New
Zealand white rabbits; one group received components with conventional HA coating, while the other group received implants with SN doped HA coatings. The controls were unused implants of both types. After 12 weeks, animals were sacrificed and implants were subjected to pull-out testing. Osseointegration of retrieved implants and resorption of SNs were examined using scanning electron microscopy (SEM) with energy-dispersive $X$-ray spectroscopy (EDS). The area of implant-bone contact was also evaluated on cross-sections of the retrieved implants.

\section{Implant preparation}

Implants used in this study were made from commercially available TiAl6V4 alloy. First, cylinders with a diameter of $4 \mathrm{~mm}$ and length of $22 \mathrm{~mm}$ were prepared, and one of their ends was threaded, while other parts were sandblasted and plasma-sprayed with a 25-30 $\mu \mathrm{m}$ layer of HA (2PS, France). Next, randomly selected implants were doped with commercially available SNs (Particular GmBH, Hannover, Germany). Briefly, implants were immersed in a suspension containing nanoparticles with a mean diameter of $66 \mathrm{~nm}$, and $\mathrm{Ag}$ concentration of $106 \mathrm{mg} / \mathrm{L}$. The liquid was stirred at room temperature for 14 hours, after that time implants were removed, washed with physiological saline and sterilized in ethylene oxide (Fig. 1A).

\section{Animal experiments}

All tests involving animals were conducted according to an approval granted by the local bioethical committee; the animals used were New Zealand white rabbits, (mean weight $4.2 \mathrm{~kg}$, range 3.0-5.5). Briefly, after animals underwent general anaesthesia (ketamine + medetomidine), their femora were exposed through a lateral incision. In each case, a 4-mm transverse hole was drilled perpendicularly to the long axis of the bone, and a randomly selected test implant was then inserted (Fig. 1B). Each animal received one implant with SNs and one control sample with conventional HA coating in the contralateral femur. All animals survived the procedure, with no complications and were then kept in cages, receiving food and water ad libitum. After 12 weeks, rabbits were euthanized (intracardiac pentobarbital injection) and the femora containing metal cylinders were immediately harvested and stored in a refrigerator for up to 48 hours. Next, pull-out testing was performed using a similar technique to that described by Salou et al. [16] using an Instrom 4483 load frame (Instrom, UK). 


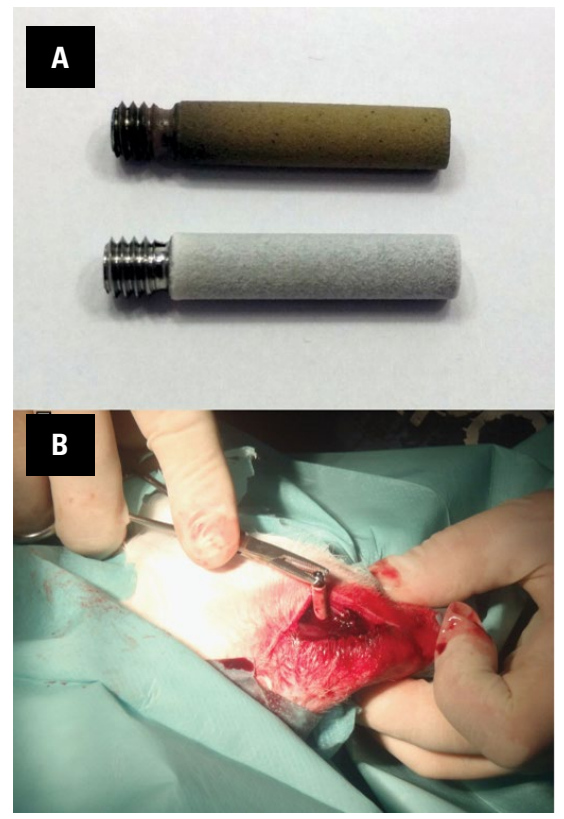

Figure 1. Animal experiments; A. Implants used for the experiment; top - implant with silver nanoparticles doped hydroxyapatite (HA) coating, bottom - implant with standard HA coating; B. Implantation of a HA-coated Ti rod into the femur of a New Zealand white rabbit, intraoperative view.

Briefly, after the specimens were warmed to room temperature the threaded part of each implant was screwed to the crosshead of the device and the femora were clamped using a custom fixture. The uniaxial test was carried with a speed of $1 \mathrm{~mm} / \mathrm{min}$, ant the maximum pull-out force was recorded for each specimen with an accuracy of $0.1 \mathrm{~N}$. After testing retrieved implants were fixed in $2.5 \%$ buffered glutaraldehyde solution for 7 days and prepared for SEM examination (Fig. 1).

\section{SEM preparation and analysis}

After fixation, retrieved samples were dehydrated in a series of ethanol/water solutions $(30,50,70,96$ and $100 \%$ ) for 12 hours. Due to concerns regarding the behaviour of the metal-coating interface during critical point drying, an alternative method of SEM preparation was performed. Similar to Araujo et al. [1], final dehydration was performed by immersion of samples in hexamethyldisilazane for 2 minutes twice, followed by air drying for 60 minutes. Most explants were sputter coated with $10 \mathrm{~nm}$ of gold (JEOL JFC1200) for SEM (JEOL JSM-6400) examination. Imaging was performed predominantly using backscatter electron detector (BSD) at 20-30 kV, since the contrast of such images is related to the elemental composition of various structures (Z-contrast mode).
Selected areas were photographed using secondary electron detector for better topographic contrast, and some samples were examined using EDS (Oxford Instruments Inca Energy) to verify their elemental composition.

\section{Quantification of bone ingrowth: bone-implant contact percentage (BIC\%)}

After microscopic examination of explants, two cross-sections (5 $\mathrm{mm}$ apart) from the area which was in direct contact with bone were prepared using a diamond wafering saw (Buehler Isomet low speed saw). They were examined using SEM to determine the percentage of implant area in direct contact with bone. Imaging was performed using BSD at $250 \times$ magnification. Micrographs were taken at a short working distance of 10-12 mm, which results in a shallow depth of field, allowing the precise determination if the attached bone was in the cross-section plane. Areas in direct contact with bone were then quantified by manual outlining using ImageJ 1.49 software. Areas where large metal shards or coating damage were present as a result of cutting (up to $10 \%$ of implant circumference) were excluded from the analysis, yielding a total of 18-22 images per cross-section, which were used to perform the statistical analysis using Statistica 13.1 software (Statsoft Inc.). First, the normality of data distribution was evaluated using the Shapiro-Wilk test, then differences between BIC\% measured on cross-sections from HA and SN samples from each animal were evaluated.

\section{RESULTS}

Microscopic examination of unused reference samples demonstrated similar morphology of the HA layers in implants with and without SNs; both coatings consisted of randomly scattered HA splats and grains forming a microporous structure (Fig. 2A, B). Silver nanoparticles (as confirmed by EDS analysis) were also randomly scattered on the surface of the implants; however, in recessed areas, they tended to form small clumps up to $1 \mu \mathrm{m}$ in diameter (Fig. 2C, D).

In all retrievals, coating integrity was not compromised after pull-out tests, indicating good bonding strength to the substrate material. In both types of samples, good osseointegration was observed with formation of trabecular bone around the implants; mean pull-out forces were 122.5 (range 87.2-152.5) and 110.4 (range 72.0-162.4) for the HA and SN groups. During these tests the macroscopic trabecu- 


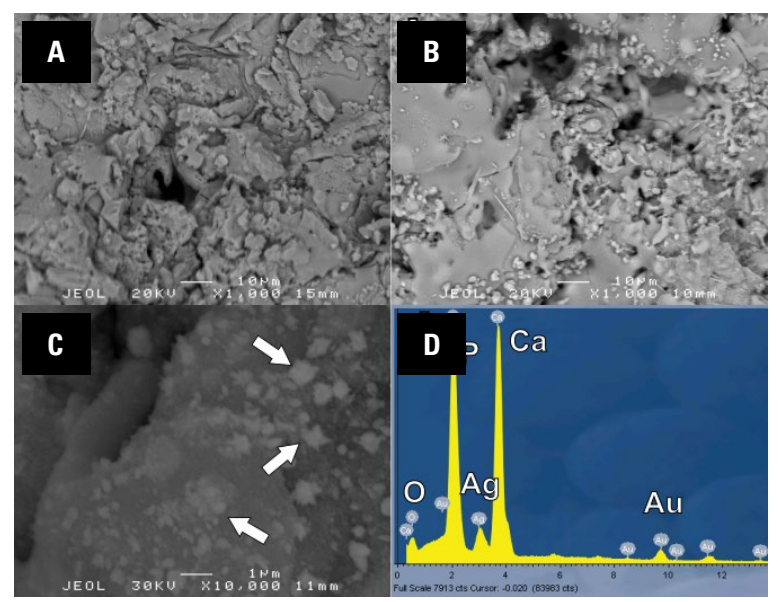

Figure 2. Scanning electron microscopy analysis of unused reference samples; A. Typical microstructure of the plasma-sprayed hydroxyapatite (HA) coating (BSD image); B. Microstructure of the HA coating with silver nanoparticles (BSD image); C. Silver nanoparticles and their conglomerates indicated by arrows (BSD image); $\mathbf{D}$. Energy-dispersive X-ray spectroscopy analysis of the nanoparticles confirming presence of silver $(\mathrm{Ag})$ on the surface of the coating (sample was sputter-coated with gold prior to analysis); 0 - oxygen; $\mathrm{P}$ - phosporus; $\mathrm{Ca}$ - calcium, $\mathrm{Au}$ — gold (sputtering material).

lar structure was damaged (Fig. 3A); however, large patches of bone were still attached to the coatings, indicating very good bonding strength of the mineral matrix. The microscopic morphology of bone attached to the coatings was identical in native HA coatings and samples doped with SNs (Fig. 3B, D). In all cases, areas covered with flat bony structures, which tended to penetrate most pores of the coating, were visible. In areas adjacent to trabeculae, there was a gradual transitional zone with a thin layer of mineralised tissue in both conventional and SN coted samples. This was clearly visible in BSD images, where mineralised bone had a lower signal intensity in comparison to the HA coating (Fig. 3C, E). In the silver doped samples, it was observed that some particles were still present after 12 weeks in vivo in areas not in direct contact with bone (Fig. 3F).

Energy-dispersive $X$-ray spectroscopy analysis was conducted to verify if the newly formed structures were indeed bony tissue. In all samples, the majority of tissue attached to the coating contained a large percentage of calcium, indicating that it was indeed mineralised bone. Smaller patches were covered by fragments composed predominantly of carbon, which suggests that these were connective tissue fragments. Identical EDS spectra were observed for soft tissue fragments attached to implant fragments not coated by HA, such as the threaded regions. SEM analysis of

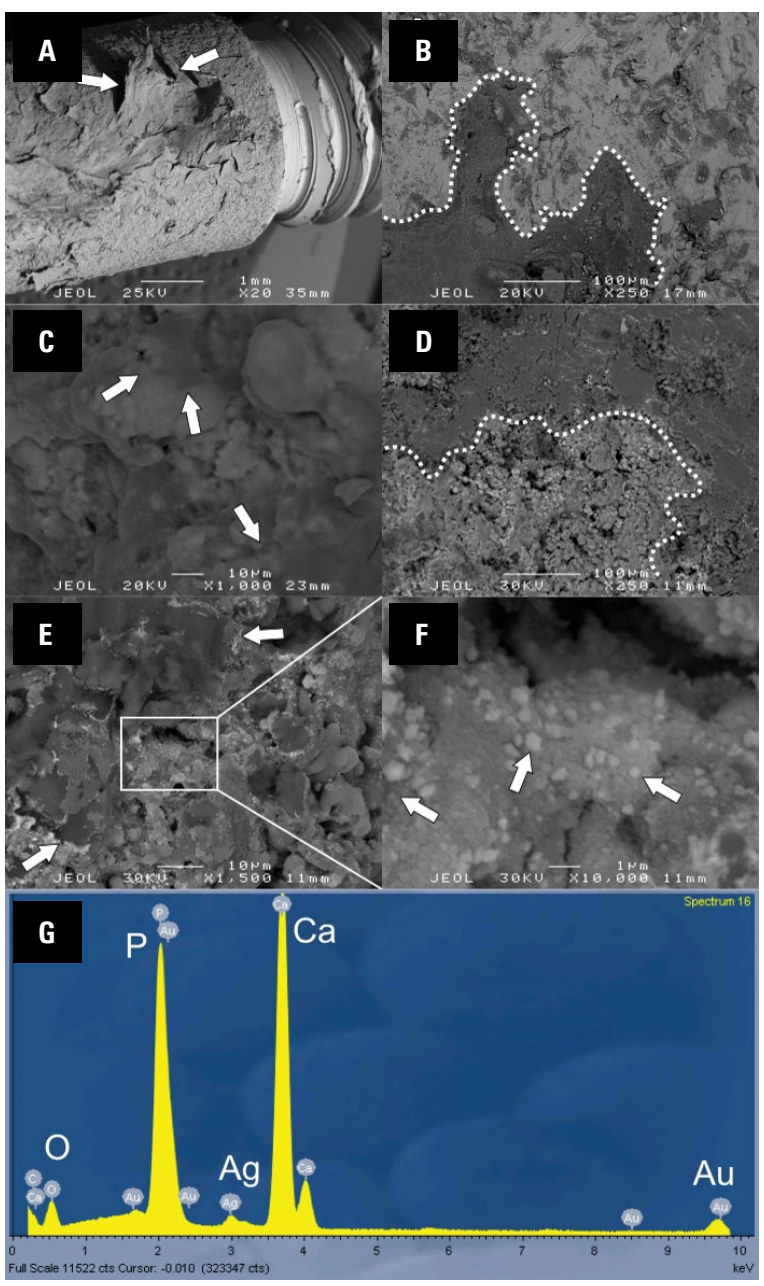

Figure 3. Retrieved implants after pull-out tests: A. Fragments of trabecular bone still attached to the retrieved component (SE image), arrows indicate partially debonded fragments; B. Ingrowth of bone (lower, dark part of micrograph) into the standard hydroxyapatite (HA) coating (BSD image); C. High magnification micrograph of newly formed mineral bone between HA grains (BSD image), arrows indicate thin mineralised layers on HA coatings; $\mathbf{D}$. Low magnification view of newly formed trabeculae (dark, upper part of micrograph) on an implant with $\mathrm{HA}+$ silver nanoparticles (SNs) coating (BSD image); E. High magnification demonstrating good ingrowth of bone into the $\mathrm{HA}$ coating with SNs (BSD image), arrows indicate thin mineralised layers of bone; F. High magnification view of central part of panel $E$, arrows indicate $\mathrm{SNs}$ attached to the HA substrate; G. Energy-dispersive $X$-ray spectroscopy analysis of $S N$ type coating $(10 \times 10 \mu \mathrm{m}$ area containing nanoparticles) from one of retrievals confirming presence of silver, (sample was sputter-coated with gold prior to analysis); $\mathrm{O}$ - oxygen; $\mathrm{P}$ - phosporus; $\mathrm{Ca}$ - calcium, $\mathrm{Ag}$ - silver; $\mathrm{Au}$ - gold (sputtering material).

cross-sections indicated that after 12 weeks, a large portion of the implant was in direct contact with trabecular bone. Also, SEM studies typically demonstrated bony fragments with a thickness exceeding $100 \mu \mathrm{m}$ firmly attached to the samples (Fig. 4A). In regions where no bone contact occurred, only a thin structure of the HA coating was visible (Fig. 4B). In all cases, the 

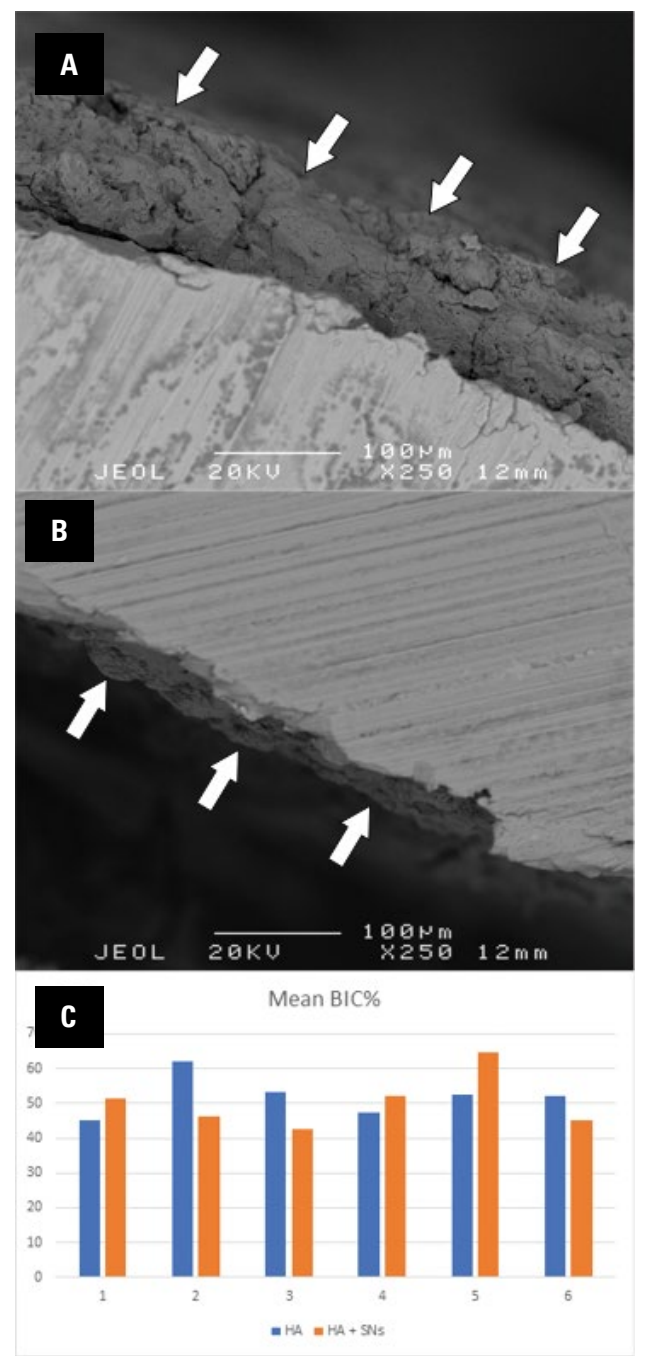

Figure 4. Bone-implant contact analysis; A. Cross-sections of an retrieved implant with mineralised bone formed on the hydroxyapatite (HA) coating (arrows, BSD image); B. Fragment of the implant with no bone attached, thin HA coating visible (arrows, BSD image); C. Mean bone-implant contact percentage $(\mathrm{BIC} \%)$ values averaged from two cross-sections in all samples examined in this study; HA - samples with hydroxyapatite coating; $\mathrm{HA}+\mathrm{SNs}$ - hydroxyapatite layer with silver nanoparticles.

bone was firmly attached to the coating, with no gaps visible at the interface. Measurements of the area of implants covered by bone indicated comparable results for both types of components. Bone implant contact area (and standard deviations) were calculated from summarised morphometric results from two cross-sections per implant (12 results per group). The mean $\mathrm{BIC} \%$ were $52.0 \pm 5.34 \%$ and $50.5 \pm$ $\pm 7.17 \%$ in implants with conventional and SN doped HA coatings respectively (Fig. 4C). There were no statistical differences between $\mathrm{BIC} \%$ measurements taken in HA and SN samples in all cases, except for case number 2, where $\mathrm{BIC} \%$ was significantly higher for the HA sample ( $p<0.05$, Wilcoxon test).

\section{DISCUSSION}

Uncemented fixation is currently the gold standard in many types of arthroplasties, predominantly in hip, shoulder and knee joints as well as modern dental implants $[5,7,15,17]$. As the number of joint replacement procedures is increasing in developed countries, management of arthroplasty related complications is becoming more important. Among the major complications following these procedures, periprosthetic joint infections are predominantly problematic in management and associated with a severe financial burden [20]. In recent years, many studies focused on possible methods of minimising the incidence of PJIs, and several authors suggested that application of SNs could be effective [1, 2, 6, 22]. Although antimicrobial properties of SNs are well documented, their effect on osteointegration of orthopaedic implants is not well understood $[3,4$, $8,21,23]$. A rabbit model was used in this study to examine a series of implants with conventional and $\mathrm{SN}$ doped HA layers and demonstrated comparable osseointegration of both coatings.

The present study has several limitations, predominantly related to the fact that it includes a small number of retrievals. This limitation is due to ethical considerations, animal studies are required to include the lowest possible number of animals, so only a small number of samples was available in this and similar studies conducted by other authors $[5,8,9]$. Another limitation is the use of pull-out testing for the evaluation of implants. This procedure did not allow samples to be obtained for light microscopy, moreover several bones fractured during these tests, compromising the quality of the obtained data. Although mechanical testing of implants was not within the scope of this study, it should be noted that pull-out forces were comparable in both groups, consequently the extent of mechanical damage to the bone-implant interface was most likely HA and SN implants. The time between bone removal and mechanical testing was relatively long, and samples were fixed after pull-out tests. Such a protocol could result in unpredictable artefacts in conventional microscopy, so the tissue samples were discarded. Nonetheless, this procedure allowed the retention of well-fixed bone trabeculae, which play a critical role in the long term performance of the implant, providing a unique in- 
sight into the bone-implant interface. In addition, a tungsten-filament SEM was used with a resolution of approximately 10-15 nm in BSD mode, which limited the precision in imaging the smallest Ag particles. The use of a field emission gun SEM with sub-nanometre resolution would be more desirable, but such equipment is costly and other studies have reported valuable data using conventional SEM $[1,13,19,21]$.

It was observed that both SN doped HA coatings allow for excellent osseointegration in vivo, comparable to that of conventional HA. Although animal models have been extensively used to verify the performance of various bone ingrowth layers, very few authors examined implants containing $\mathrm{Ag}$ nanoparticles $[8,9,23]$. In the case of rabbits, only three studies have been conducted so far, and focused on optical microscopic examination. These papers confirmed good osseointegration at 10-12 weeks, did not demonstrate local or systematic toxicity, and indicated antimicrobial effectiveness of SNs. Data from laboratory studies indicated comparable adhesion and proliferation of osteoblasts on both types of materials, and similar release of osteoinductive mediators $[3,4,8,17,21,23]$. The present study expanded this knowledge by demonstrating that the morphology of the interfacial zone is identical for both types of material. SEM observations indicated a comparable mechanism of new bone formation and mineralisation, and there was no evidence suggesting that SNs would retard or prevent formation of trabeculae. Similar new bone morphology was reported in other studies in which samples were subjected to pull-out testing $[3,4,7,13]$.

Data from this quantitative examination suggests that the presence of $\mathrm{Ag}$ nanoparticles does not affect bone formation at the macroscopic scale, since bone-implant contact areas were similar in both groups. There was a smaller $\mathrm{BIC} \%$ in this study than those reported for other uncemented implants not subjected to pull-out testing and were conducted using light [4] or electron microscopy [10, 16]. It is possible that the testing procedure may have removed some of the attached trabeculae, which may have lowered the $\mathrm{BIC} \%$. We believe that the electron microscopy preparation procedure did not influence the results in a significant way. Some authors used critical point drying to dehydrate of samples [18] subjected to pull-out testing prior to SEM analysis. Such procedure is associated with rapid changes of temperature and pressure, which could potentially result in cracking and delamination at the interfaces between bone, coating and implant due to differences in their physical properties such as thermal expansion coefficients. We therefore decided to perform dehydration by immersion in hexamethyldisilazane, which eliminates rapid pressure gradients, and does not deteriorate the quality of samples as fragile as biofilms [1]. Still, it should be underlined, that the significance of quantitative analysis is limited, since a meaningful statistical analysis was not possible due to the small sample size. Additionally only two cross-sections were examined per implant, since the remainder was used in other, destructive type tests. This limits the precision of our measurements; however, it should be noted that other authors typically performed measurements using one cross-section.

To our knowledge, this is the first study to demonstrate that Ag nanoparticles can be retained on the surface of bone implants for as long as 12 weeks. Resorption of SNs has been studied in various laboratory and in vivo settings, and it has been suggested that they can remain active for up to several weeks $[14,22]$. The present findings indicate that in the case of bone implants, SNs may be retained longer, especially given the fact that no degradation was observed. This is potentially important for clinical practice, as it suggests that the protective role of SNs may extend beyond the early postoperative period. Although this study used X-ray spectroscopy to confirm the atomic composition of various structures, this technique could not be used to conduct quantitative analyses of silver retention in the doped samples due to the presence of heterogeneous layers of bone in the samples. Differences in local thickness would significantly affect the results of EDS analysis. Moreover, this type of spectroscopy has limited precision $(1-3 \%)$, so only qualitative results are presented $[2,7,11,13,19,21,23]$.

\section{CONCLUSIONS}

The present study indicates that HA coatings doped with SNs have good osseointegrative properties, comparable to those of conventional HA, making them potentially suitable for use in orthopaedic implants. The Ag nanoparticles may be retained at the bone-implant interface for as long as 12 weeks, suggesting a prolonged antimicrobial effect. Further studies investigating multiple nanoparticle parameters, such as size, number and technique used for bonding with $\mathrm{HA}$, should be conducted to fine-tune 
the composition of SN doped coatings for optimal osseointegrative and antimicrobial properties.

\section{Acknowledgements}

This study was supported by a European Union grant no. 2204/7.PR/2011/2 "Nanomining".

\section{REFERENCES}

1. Araujo JC, Téran FC, Oliveira RA, et al. Comparison of hexamethyldisilazane and critical point drying treatments for SEM analysis of anaerobic biofilms and granular sludge. J Electron Microsc (Tokyo). 2003; 52(4): 429-433, indexed in Pubmed: 14599106.

2. Besinis A, Hadi SD, Le HR, et al. Antibacterial activity and biofilm inhibition by surface modified titanium alloy medical implants following application of silver, titanium dioxide and hydroxyapatite nanocoatings. Nanotoxicology. 2017; 11(3): 327-338, doi: 10.1080/17435390.2017. 1299890, indexed in Pubmed: 28281851.

3. Bjursten LM, Rasmusson L, Oh S, et al. Titanium dioxide nanotubes enhance bone bonding in vivo. J Biomed Mater Res A. 2010; 92(3): 1218-1224, doi: 10.1002/jbm.a.32463, indexed in Pubmed: 19343780.

4. Cheng $Y$, Gao Bo, Liu X, et al. In vivo evaluation of an antibacterial coating containing halogenated furanone compound-loaded poly(l-lactic acid) nanoparticles on microarc-oxidized titanium implants. Int J Nanomedicine. 2016; 11: 1337-1347, doi: 10.2147/IJN.S100763, indexed in Pubmed: 27099494.

5. Chisnoiu R, Moldovan M, Păstrav O, et al. The influence of three endodontic sealers on bone healing: an experimental study. Folia Morphol. 2016; 75(1): 14-20, doi: 10.5603/ FM.a2015.0079, indexed in Pubmed: 26383505.

6. Ciobanu CS, Iconaru SL, Chifiriuc MC, et al. Synthesis and antimicrobial activity of silver-doped hydroxyapatite nanoparticles. Biomed Res Int. 2013; 2013: 916218, doi: $10.1155 / 2013 / 916218$, indexed in Pubmed: 23509801

7. Giavaresi G, Fini M, Cigada A, et al. Mechanical and histomorphometric evaluations of titanium implants with different surface treatments inserted in sheep cortical bone. Biomaterials. 2003; 24(9): 1583-1594, indexed in Pubmed: 12559818.

8. Kose N, Çaylak R, Pekşen C, et al. Silver ion doped ceramic nano-powder coated nails prevent infection in open fractures: In vivo study. Injury. 2016; 47(2): 320-324, doi: 10.1016/j.injury.2015.10.006, indexed in Pubmed: 26589596

9. Kose N, Otuzbir A, Pekşen C, et al. A silver ion-doped calcium phosphate-based ceramic nanopowder-coated prosthesis increased infection resistance. Clin Orthop Relat Res. 2013; 471(8): 2532-2539, doi: 10.1007/s11999-0132894-x, indexed in Pubmed: 23463287.

10. Miura K, Yamada N, Hanada S, et al. The bone tissue compatibility of a new Ti-Nb-Sn alloy with a low Young's modulus. Acta Biomater. 2011; 7(5): 2320-2326, doi: 10.1016/j. actbio.2011.02.008, indexed in Pubmed: 21316491.

11. Morones JR, Elechiguerra JL, Camacho A, et al. The bactericidal effect of silver nanoparticles. Nanotechnology. 2005;
16(10): 2346-2353, doi: 10.1088/0957-4484/16/10/059, indexed in Pubmed: 20818017.

12. Nirmala R, Sheikh F, Kanjwal M, et al. Synthesis and characterization of bovine femur bone hydroxyapatite containing silver nanoparticles for the biomedical applications. J Nanopart Res. 2010; 13(5): 1917-1927, doi: 10.1007/ s11051-010-9944-z.

13. Ogawa T, Ozawa S, Shih JH, et al. Biomechanical evaluation of osseous implants having different surface topographies in rats. J Dent Res. 2000; 79(11): 1857-1863, doi: 10.1177/ 00220345000790110701 , indexed in Pubmed: 11145355.

14. Osibe DA, Chiejina NV, Ogawa K, et al. Stable antibacterial silver nanoparticles produced with seed-derived callus extract of Catharanthus roseus. Artif Cells Nanomed Biotechnol. 2018; 46(6): 1266-1273, doi: 10.1080/2169140 1.2017.1367927, indexed in Pubmed: 28830244.

15. Punke Ch, Zehlicke T, Just $T$, et al. Matrix change of bone grafting substitute after implantation into guinea pig bulla. Folia Morphol. 2012; 71(2): 109-114, indexed in Pubmed: 22648590.

16. Salou L, Hoornaert A, Louarn G, et al. Enhanced osseointegration of titanium implants with nanostructured surfaces: an experimental study in rabbits. Acta Biomater. 2015; 11: 494-502, doi: 10.1016/j.actbio.2014.10.017, indexed in Pubmed: 25449926.

17. Saravanan S, Nethala S, Pattnaik $S$, et al. Preparation, characterization and antimicrobial activity of a bio-composite scaffold containing chitosan/nano-hydroxyapatite/nano-silver for bone tissue engineering. Int J Biol Macromol. 2011; 49(2): 188-193, doi: 10.1016/j.ijbiomac. 2011.04.010, indexed in Pubmed: 21549747.

18. Simmons CA, Valiquette N, Pilliar RM. Osseointegration of sintered porous-surfaced and plasma spray-coated implants: An animal model study of early postimplantation healing response and mechanical stability. J Biomed Mater Res. 1999; 47(2): 127-138, indexed in Pubmed: 10449624.

19. Sorkun HÇ, Yay AH, Balcıoğlu E, et al. Assessment of femoral neck fractures in the elderly with respect to morphology and mineral density. Folia Morphol. 2016; 75(4): 536-542, doi: 10.5603/FM.a2016.0013, indexed in Pubmed: 27830871.

20. Vanhegan IS, Malik AK, Jayakumar $P$, et al. A financial analysis of revision hip arthroplasty: the economic burden in relation to the national tariff. J Bone Joint Surg Br. 2012; 94(5): 619-623, doi: 10.1302/0301-620X.94B5.27073, indexed in Pubmed: 22529080.

21. van Hengel IAJ, Riool M, Fratila-Apachitei LE, et al. Selective laser melting porous metallic implants with immobilized silver nanoparticles kill and prevent biofilm formation by methicillin-resistant Staphylococcus aureus. Biomaterials. 2017; 140: 1-15, doi: 10.1016/j.biomaterials.2017.02.030, indexed in Pubmed: 28622569.

22. Wu D, Fan W, Kishen A, et al. Evaluation of the antibacterial efficacy of silver nanoparticles against Enterococcus faecalis biofilm. J Endod. 2014; 40(2): 285-290, doi: 10.1016/j. joen.2013.08.022, indexed in Pubmed: 24461420.

23. Xie CM, Lu X, Wang KF, et al. Silver nanoparticles and growth factors incorporated hydroxyapatite coatings on metallic implant surfaces for enhancement of osteoinductivity and antibacterial properties. ACS Appl Mater Interfaces. 2014; 6(11): 8580-8589, doi: 10.1021/am501428e, indexed in Pubmed: 24720634. 